\title{
Content-Based Navigation Within Virtual Museums
}

\author{
Anestis Koutsoudis, Christina Makarona and George Pavlidis \\ 'ATHENA' Research and Innovation Center, Xanthi, GR-67100, Greece \\ e-mails: \{akoutsou; cmakar; gpavlid\}@ ceti.gr
}

\begin{abstract}
The ongoing evolution of the Internet has enabled web applications that use real time three-dimensional (3D) graphics and virtual environments. Moreover, in recent years, cultural technology has also flourished notably. Virtual museums represent one significant application that combines culture with modern technologies and the internet, requiring an interdisciplinary approach aiming both at dissemination and education. We present our approach to enhance virtual museums by incorporating content-based retrieval that refers to $3 \mathrm{D}$ objects. In this context, a virtual visitor is able to navigate within the museum, examine the exhibited artifacts and perform queries-byexample in order to locate the position of related artifacts. The retrieval mechanism responds to the visitor's queries by indicating similar artifacts, which are ranked according to their similarity scores. This way, the visitor is able to navigate within a virtual museum not only by means of a virtual walkthrough but also by means of the context.
\end{abstract}

Keywords: $3 D$ content based retrieval, $3 D$ pottery shape matching, virtual museum, virtual reality.

\section{Introduction}

Modern archaeology utilizes technologies such as 3D digitization and computer graphics to improve its research methods. The digitization of artifacts applies not only to cultural preservation but also to the dissemination, management, smart indexing and retrieval. As several digitization projects have been crowned with success, the production of numerous 3D digital archives is inevitable. 3D content based retrieval (3DCBR) is an advanced data indexing and management mechanism that can be used to automate the identification of shape similarities 
within 3D digital archives. Most of the experimental 3DCBR systems found in literature, follow the traditional thumbnail-image tiling visualization approach for presenting query results [1]-[11]. A 2D thumbnail render is used instead of the actual 3D model in order to reduce the amount of data being transferred. The query results are presented ordered according to similarity. On the other hand, for certain domains, the provision of a 3D environment allows the visualization to be closer to reality. This is true for applications with inherent 3D spatial properties, e.g. archaeological, architectural or geological data [12]. Broadband Internet connections and 3D graphics accelerators allow the enhancement of graphical user interfaces with technologies able to depict realistic 3D environments and support a wider set of human-computer interaction functionalities. The use of a 3D environment for data visualization and information browsing, results in a more natural and efficient, in terms of data access, graphical user interface [12]-[17]. Within this virtual world the visitor can freely navigate and change position, orientation and viewpoint direction. Additionally, such an interface relieves the visitor from the focus-plus-context problem [18]. This is true for a 3D environment as the visitor can focus on an individual 3D object and interact with it without losing the overall context of the environment in which it is being displayed or in which it belongs [18].

In this paper, we present the idea of integrating a 3DCBR system with an online non-immersive virtual reality environment. A virtual museum that exhibits pottery artifacts was implemented as a case study. The visitor can browse a collection of $3 \mathrm{D}$ vessels in a more natural way and perform queries-by-example by selecting any of the virtual exhibits. An interactive map that contains a top-view of the museum allows the user to identify his/her position in the museum and also to review the spatial and ranking positions of the query results. The content of the museum is dynamic in a sense that all exhibits are retrieved from a database. The current implementation can be accessed through a Web browser and was built both on open and commercial software technologies.

\section{Web-Based 3D Environments}

Web-based virtual reality environments have already been utilized by several researchers for the visualization of texts, images, audio and 3D data. In [14] a 3D content-based visualization environment was introduced, using the metaphor of a universe to present different categories of traditional Greek music and provide access to the content itself, based on clustering guided by audio descriptors and neural networks. Tian et al. [19] presented a content based retrieval system for digital images which organizes the query results in a 3D space. Ciocca et al. [20] implemented a Web-based system for interactive search of items in electronic catalogues. A subset of images in which the user was interested was presented in a 3D virtual exhibition using VRML. Nakazato et al. [21] worked on a similar system which was implemented in an immersive virtual reality CAVE visualization system. Assfalg et al. [22] proposed a 3D environment which could 
be customized by adding simple 3D objects and editing them in terms of positioning, scaling, colors and textures. The system allowed the user to freely navigate within the 3D scene and capture a screenshot of it which could be used as a query-by-example to an image database. Wang et al. [23] proposed a sophisticated 3D visualization layout for query results derived from an image database. The proposed approach maximized the entropy of the presented information. Similarly, Bonnel et al. [24] focused on processing the query results of a textual search engine in order to effectively organize and visualize them using a 3D city metaphor environment. On the other hand, Colaci et al. [25] presented a tool for analysis and classification of 3D VRML scenes. They designed a system for 3D scenes which contained furnished environments and a set of human avatars. By exploiting the hierarchical structure of the VRML scene the system extracted all objects and responded to the user with a textual analysis of the scene's content. Andreou et al. [26] described a system which allowed the creation of novel 3D scenes by incorporating models included in a database. The user could sketch an object in two dimensions and use it as a query-by-example. Ballegooij et al. [27] presented the idea of navigation-by-query in a 3D environment in order to overcome the classic lost-in-cyberspace problem experienced in geometrically complex 3D virtual environments.

Several researchers have proposed the use of real time 3D graphics technologies for the implementation of Web-based virtual museums. Shiaw et al. [18] presented an interactive 3D museum of Greek pottery, where the user could navigate from a high level scatter plot-like plan view to a perspective overview of a subset of a pottery collection using VRML technologies. Pavlidis et al. [28] described a technological framework for a virtual museum which exhibited 3D replicas of musical instruments. Patel et al. [29] presented a system designed to create 3D virtual exhibitions of museum artifacts. Isler et al. [30] described a similar system focused on 3D digital replicas of Native American baskets. Recently, Fominykh et al. [31] presented a 3D virtual museum which incorporates collaborative technologies in order to serve as a virtual workshop for educational purposes. Charitos et al. [32] discussed the advantages of installing and using a virtual museum system within a real museum. Oberlander et al. [33] described the implementation of a virtual gallery of artifacts based on the Second Life 3D game engine.

\section{Description of The Proposed System}

The proposed system integrates the functionality of a 3D content based retrieval mechanism with a dynamic Web-based interactive 3D environment, producing an integration that enhances the visitors' experience, as the system can automatically respond to questions such as 'Where else in the museum can I find artifacts similar to this one?' or 'Which one is the most similar artifact to this one?'. 
Fig. 1 depicts the overall technological framework under consideration. On one hand, there is the 3D data repository, produced either by 3D digitization or by $3 \mathrm{D}$ modeling. There is a content-based analysis system that extracts 3D shape features, which, along with documentation data and metadata form the complete data source. On the other hand there is the web-based virtual museum visualization and user interaction system, along with the querying system, which enables the execution of similarity-based requests.

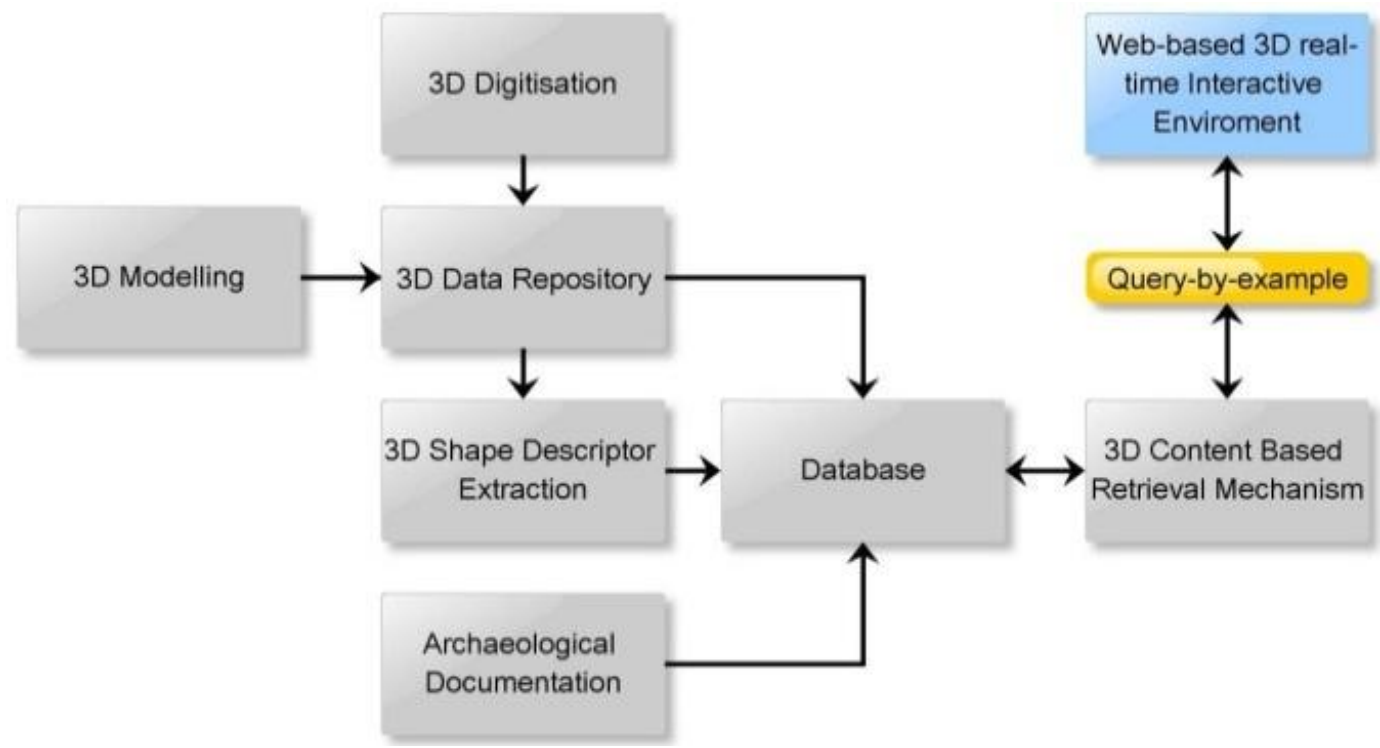

Fig. 1. The overall proposed technological framework

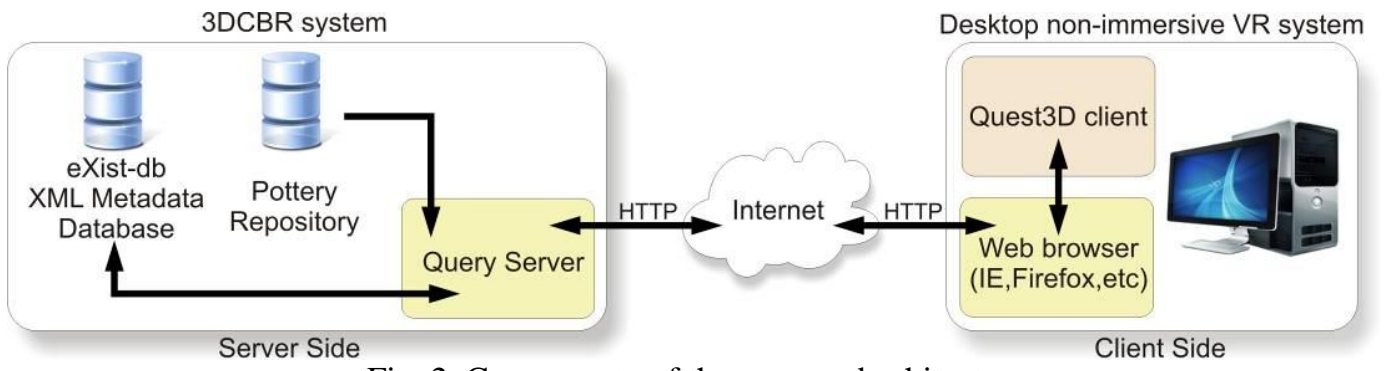

Fig. 2. Components of the proposed achitecture

As shown in Fig. 2, the system is based on the client-server architecture. Both sides can be implemented using various available technologies either open or commercial. In this work, we have adopted a hybrid approach involving technologies from both sectors:

- The server side is composed by the query server, the metadata database and the repository. The server side is implemented using open source technologies, with the query server being implemented in PHP and the database in native XML eXist database. The database includes metadata contain information related to basic cultural information (shape, type of shape, attribution, 
workshop, preservation state, etc.) [34], the 3D shape descriptor used for shape matching (our previously published 3D shape matching descriptor [2] and a set of predefined 3D coordinates, which are used to position the exhibits within the virtual museum. The query server is responsible for populating the virtual museum in the client side with the artifacts and their metadata. Additionally the query server handles all queries-by-example and forwards the similarity ranking scores to the client. Furthermore, as the repository contains Web-oriented copies of the 3D exhibits (stored in a custom DirectX file format, which incorporates 3D meshes and bitmap texture maps in a single file).

- In the client side there is supposed to be a Web-browser equipped with a $3 D$ graphics plug-in that will allow the visitor's browser to display the virtual museum and to perform queries by example using the exhibits within the museum.

During a virtual visit, a user can freely navigate within the different galleries of the museum. A first-person game approach is adopted where collision detection does not apply to exhibits (the digital replicas are relieved from the 'Do not touch' tag). The visitor can focus on an artifact, get information about it and arbitrarily rotate it. The visitor can also select an exhibit and submit a query-by-example. The system replies to queries by dynamically updating the content of the 3D environment. Animated arrows appear over the relevant exhibits in order to point out their positions in the museum. An interactive map is used to depict a top-view of the museum with real-time indication of the visitors position and direction, along with the positions of relevant exhibits and their similarity scores, assisting the visitor to avoid any of the lost-in-cyberspace situations.

The overall system communication is as follows: during the initialization the client requests from the server the 3D model of the virtual museum. Once the model is downloaded, the client automatically submits a query requesting the museum exhibits (3D models and metadata). The server replies by providing metadata that contain information regarding the exhibit's unique ID, textual information about the exhibit, a link to the Web-oriented 3D model and the predefined spatial coordinates of the exhibit that will be used to position it in the appropriate collection inside the museum. The client uses this information to progressively populate the galleries of the virtual museum. After the completion of the initialization phase, the client is ready to forward queries-by-example to the 3DCBR system. Once an exhibit is selected as a query object, the client submits a query to the server using the exhibit's ID. The query server performs shape matching by computing the morphological similarity between the query object and the objects in the database [34]. Once the similarity ranking scores are computed, they are returned sorted to the client. The client interprets the similarity scores and updates the content of the 3D environment. As the server returns the similarity scores for all vessels in the database, a score threshold is used to limit 
the number of animated arrows that appear as relevant exhibits indicators, preventing the appearance of indicators over low similarity exhibits. The results of a query remain visible until a new query is submitted to the server.

In order to test the proposed idea, we have implemented a Web-based 3D virtual museum that exhibits 3D vessel replicas. The repository consists of 61 vessels, 6 of them being the product of 3D digitization while the other being manually modeled. The 3D model of the museum was initially built using Blender and was imported in Quest3D, where the whole functionality of the virtual museum was developed. It has also been used for the initial positioning of the vessels in the museum's showcases. As the environment is based on Quest3D, the client side requires the Quest3D browser plug-in. Fig. 3 shows a few screenshots of the virtual museum in action.

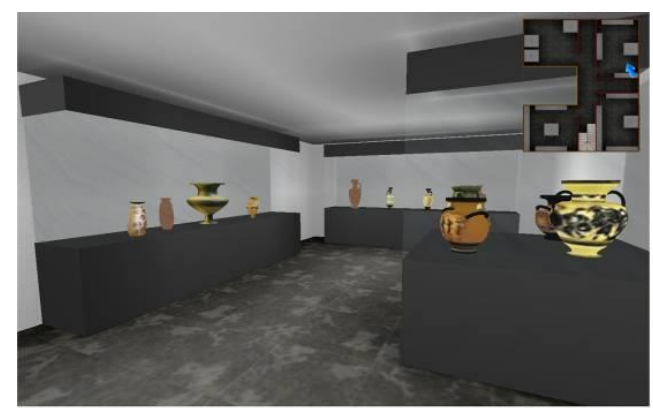

a) General view of a gallery

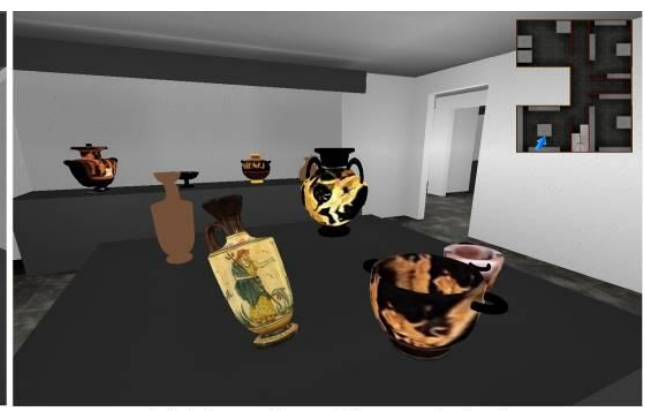

b) Interacting with an artefact

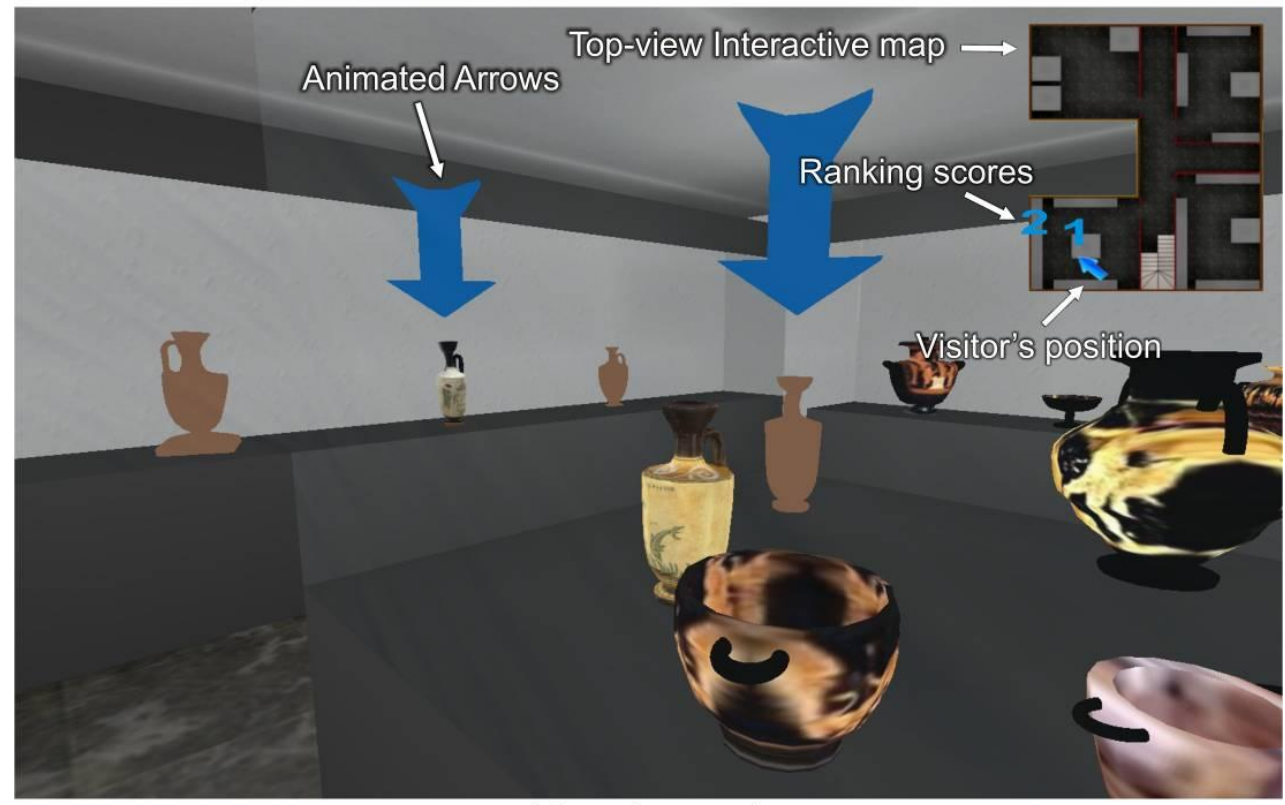

c) Query-by-example

Fig. 3. Screenshots from the 3D pottery virtual museum 


\section{Conclusion}

By augmenting Web-based interactive 3D environments with 3D content-based retrieval mechanisms a user's experience can be significantly enhanced as similarity-based coherence in shape and color is often the case. A prototype Webbased virtual museum of 3D pottery enhanced with a 3D content based retrieval mechanism has been implemented as a case study. Within the environment a visitor can freely navigate and perform queries-by-example. Although we have implemented a case study regarding virtual museums, the proposed integration can be easily exploited in 3D reconstructions of monuments, urban areas with important architecture, facade features, etc. In addition, any of the proposed system components could also be exchanged with other in order to suit the needs of specific problems. This could include other 3D feature extraction mechanisms and other $3 \mathrm{D}$ environments, as long as the main concept of the proposed technique remains unchanged.

\section{References}

[1] J. Chen, L. McGinty, N. O'Connor, A search engine for 3D models of museum artefacts, Proceedings of the 2nd International Workshop on Personalized Access to Cultural Heritage, Hannover, Germany, 28 July - 1 August, 2008.

[2] A. Koutsoudis, G. Pavlidis, V. Liami, D. Tsiafaki, C. Chamzas, 3D pottery content based retrieval based on pose normalisation and segmentation, Elsevier Journal of Cultural Heritage, Vol. 11 (3), Jul.-Sep. 2010, pp. 329338.

[3] T. Funkhouser, P. Min, M. Kazhdan, J. Chen, A. Halderman, D. Dobkin, A Search engine for 3D models, ACM Trans. on Graphics, Vol. 22 (1), 2003.

[4] J. Rowe, A. Razdan, D. Collins, S. Panchanathan, A 3D digital library system : Capture, analysis, query and display, Proceedings of the 9th International conference on 3D Web Technology, 9-12 March, 2003, Saint Malo, France.

[5] E. A. Lmaati, A. E. Oirrak, M. N. Kaddioui, A visual similarity-based 3D search engine, Journal of Data Science, Vol. 8, 2009.

[6] D. Saupe , D. V. Vranic, 3D model retrieval with spherical harmonics and moments, Proceedings of the 23rd DAGM-Symposium on Pattern Recognition, Munich, Germany, September 12-14, 2001, pp. 392-397.

[7] D.V. Vranic, D. Saupe, 3D shape descriptor based on 3D Fourier transform, Proceedings of the EURASIP Conference on Digital Signal Processing for Multimedia Communications and Services (ECMCS), Budapest, Hungary, September 2001, pp. 271-274.

[8] C. Zhang, T. Chen, Indexing and retrieval of 3D models aided by active learning, Proceedings of the 9th ACM International conference on Multimedia, Ottawa, Canada, October, 2001. 
[9] M. Zhou, Q. Huo, G. Geng, X. Liu, A new 3D model retrieval method with building blocks, International Journal of Computer Games Technology, Vol. 2009.

[10] C.T. Kuo, S.C. Cheng, 3D model retrieval using principal plane analysis and dynamic programming, Pattern Recognition, Vol. 40 (2), 2007, 742-755.

[11] E. Onasoglou, A. Mademlis, D. Katsikas, P. Daras, M. G. Strintzis, A novel prototype for documentation and retrieval of 3D objects, Proceedings of the 14th International Conference of Image Analysis and Processing-Workshops, Modena, Italy, September 10-13, 2007, pp.89-94.

[12] J. Kennedy, K. Mitchell, P. Barclay, B. Marshall, 3D information visualisation: identifying and measuring Success, Proceedings of the 2nd International FADIVA workshop, Glasgow, Scotland, UK, 1995.

[13] J. Assfalg, A. Del Bimbo, P. Pala, Virtual reality for image retrieval, Journal of Visual Languages and Computing, Vol. 11, 2000, 105-124.

[14] G. Pavlidis, D. Tsiafakis, F. Arnaoutoglou, K. Balla, C. Chamzas, Musical universe, Proceedings of the 3rd International Conference of Museology, Mytilene, Greece, June 5-9, 2006.

[15] J. Kennedy, K. Mitchell, P. Barclay, B. Marshall, 3D information visualisation: identifying and measuring success, Proceedings of the 2nd International FADIVA workshop, Glasgow, Scotland, UK, 1995.

[16] M. Nakazato, T. S. Huang, 3D MARS: Immersive virtual reality for contentbased image retrieval, Proceedings of the IEEE International Conference on Multimedia and Expo, 2001, Tokyo, Japan, August 22 - 25, pp. 44-47.

[17] E. Gonzales, A. Evans, S. Gonzales, J. Ababia, J. Blat, Real-time visualisation and browsing of a distributed video database, Proceedings of the International Conference on Advances in Computer Entertainment Technology, Athens, Greece, October 29-31, pp. 423-424.

[18] H. Shiaw, R. J.K. Jacob, G. R. Crane, The 3D vase museum : A new approach to context in a digital library, Proceedings of the 4th ACM/IEEE Joint Conference on Digital Libraries, Tucson, Arizona, June 7-11, 2004.

[19] G. Y. Tian, D. Taylor, Colour image retrieval using virtual reality, Proceedings of the IEEE International Conference on Information Visualisation, London, UK, July 19-21, 2000.

[20] G. Ciocca, R. Schettini, A content-based retrieval system with a customizable 3D output visualizer, Proceedings of the 1st International Symposium on 3D Data Processing Visualisation and Transmission, Padova, Italy, June 19-21, 2002.

[21] M. Nakazato, T. S. Huang, 3D Mars: Immersive virtual reality for contentbased image retrieval, Proceedings of the International Conference of Multimedia and Expo, ICME 2001, Tokyo, Japan, August 22-25, 2001.

[22] J. Assfalg, A. Del Bimbo, P. Pala, Querying by photographs: Using virtual reality for content-based image retrieval, Multimedia Computing and Systems, Vol.1, 1999, 556-561. 
[23] R. Wang, S. J. McKenna, J. Han, High-entropy layouts for content-based browsing and retrieval, Proceedings of the ACM International Conference on Image and Video Retrieval, Santorini, Greece, July 8-10, 2009.

[24] N. Bonnel, V. Lemaire, A. Cotarmanac'h, A. Morin, Effective organization and visualisation of web search results, Proceedings of the 24th International Multi-Conference Internet and Multimedia Systems and Applications, Inssbrunk, Austria, February 13-15, 2006, pp. 209 - 216.

[25] R. Colaci, M. Schaerf, ICARUS: Intelligent content-based retrieval of 3D scene, Proceedings of the 12th Innovative Applications of Artificial Intelligence Conference, Austin, Texas, USA, August 1-3, 2000.

[26] I. Andreou, N.M. Sgouros, Shape-based retrieval of 3D models in scene synthesis, Proceedings of the International Conference on Systems, Man and Cybernetics,

[27] A. Ballegooij, A. Eliens, Navigation by query in virtual worlds, Proceedings of Web3D, Paderbon, Germany, February 19-22, pp. 77-83.

[28] G. Pavlidis, D. Tsiafakis, G. Provopoulos, S. Chatzopoulos, F. Arnaoutoglou, C. Chamzas, MOMI: A dynamic and Internet-based 3D virtual museum of musical instruments, Proceedings of the 3rd International Conference on Museology, Mytilene, Greece, June 5-8, 2006.

[29] M. Patel, M. White, K. Walczak, P. Sayd, Digitisation to presentation building virtual museum exhibitions, Proceedings of Vision, Video and Graphics, University of Bath, Bath, England, 2003, pp. 1-8.

[30] V. Isler, B. Wilson, R. Bajcsy, Building a 3D virtual museum of Native American baskets, Proceedings of the 3rd International Symposium on 3D Data Processing, Visualization and Transmission, University of North Carolina, Chapel Hill, USA, June 14-16, 2006, pp. 954-961.

[31] M. Fominykh, E. Prasolova-Forland, M. Morozov, From 3D virtual museum to 3D collaborative virtual workshop, Proceedings of the 8th IEEE international conference on Advanced Learning Technologies, Santander, Cantabria, July 1-5, 2008, pp. 443-445.

[32] D. Charitos, G. Lepouras, C. Vassilakis, V. Katifori, A. Charissi, L. Halatsi, Designing a virtual museum within a museum, Proceedings of the Virtual Reality, archaeology and cultural heritage conference, Glyfada, Greece, November, 2001, pp. 284.

[33] J. Oberlander, G. Karakatsiotis, A. Isard, I. Androutsopoulos, Building an adaptive museum gallery in Second Life, Proceedings of the Museum and the Web conference, Montreal, Quebec, Canada, 2008.

[34] A. Koutsoudis, G. Pavlidis, F. Arnaoutoglou, D. Tsiafaki, C. Chamzas, Qp: A tool for generating 3D models of ancient Greek pottery, Journal of Cultural Heritage, Vol. 10 (2), 2009, 281-295. 